\title{
$\cos (-920.8142--8$
}

LBL-32221

UC-414

\section{Lawrence Berkeley Laboratory UNIVERSITY OF CALIFORNIA}

\section{Accelerator \& Fusion Research Division}

Presented at the Fourteenth International Free Electron Laser Conference, Kobe, Japan, August 23-28, 1992, and to be published in the Proceedings

Physically Transparent Formulation of a Free-Electron Laser in the Linear Gain Regime

W.A. Barletta, A.M. Sessler, and L.-H. Yu

August 1992

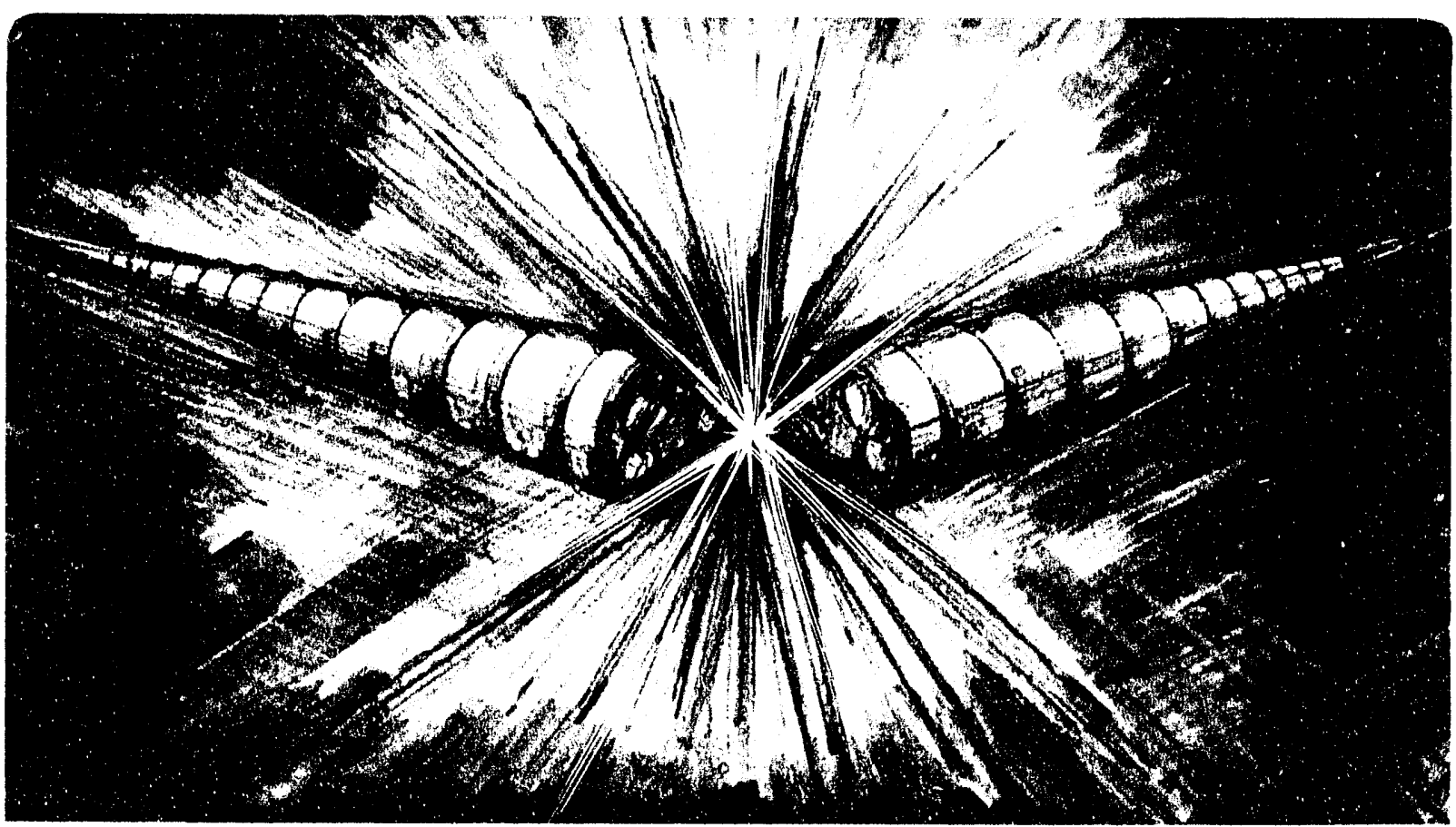

Prepared for the U.S. Department of Energy under Contract Number DE-AC03-76SF00098 
This document was prepared as an account of work sponsored by the United States Government. Neither the United States Government nor any agency thereof, nor The Regents of the University of Califo:nia, nor any of their employees, makes any warranty, express or implied, or assumes any legal liability or responsibility for the accuracy, completeness, or usefulness of any information, apparatus, product, or process disclosed, or represents that its use would not infringe privately owned rights. Reference herein to any specific commerclal product, process, or service by its trade name, trademark, manufacturer, or otherwise, does not necessarily constitute or imply its endorsement, recommendation, or favoring by the United States Govemment or any agency thereof, or The Regents of the University of California. The views and opinions of authors expressed herein do not necessarily state or reflect those of the United States Governmenit or any agency thereof or The Regents of the University of Callfornia and shall not be used for advertising or product endorsement purposes.

Lawrence Berkeley Laboratory is an equal opportunity employer.

This report has been reproduced directly from the best available copy. 
LBL -32221

DE93 002577

\title{
Physically Transparent Formulation of a Free-Electron Laser in the Linear Gain Regime
}

\author{
William A. Barletta" \\ Lawrence Livermore National Laboratory, University of California, \\ Livermore, California 94550 \\ Andrew M. Sessler" \\ Lawrence Berkeley Laboratory, University of California, \\ Berkeley, California 94720 \\ Li-Hua Yu*** \\ Brookhaven National Laboratory, Upton, NY 11973
}

August 1992

\footnotetext{
- Work supported by the Office of Energy Research, U.S. Dept. of Energy, under Contract No W-7405-eng-4's.

* Work supported by the Director, Office of Energy Research, Office of High Energy and Nuclear Physics, Division of High Energy Physics, of the U.S. Department of Energy under Contract No. DE-AC03-76SF00098.

4** Work supported by the U.S. Dept. of Energy under Contract No. DE-AC02-76CH00016
}

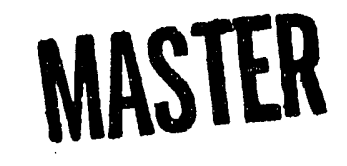




\title{
Physically Transparent Formulation of a Free-Electron Laser in the Linear Gain Regime
}

\author{
William A. Barletta ${ }^{1}$, Andrew M. Sessler ${ }^{2}$ and Li-Hua $\mathrm{Yu}^{3}$
}

\begin{abstract}
The recent 2-dimensional analytic theories of a free-electron laser (FEL) in the linear regime are reformulated in terms of three dimensionless ratios that describe the degree to which the characteristics of the electron beam deviate from the cold beam limit of a beam with no emittance or energy spread. In terms of these ratios, algebraic model equations of a fit that combines features of both of the 2-dimensional analyses are given as a convenient computational tool. Graphs of the FEL gain eigenvalue computed with the combined 2-D formulation illustrate that the gain and the output power at saturation are reduced from the 1-D value, when any of the ratios is larger than unity.
\end{abstract}

\section{Introduction}

In recent years Yu, Krinsky, and Gluckstern [1] (the BNL group) and Chin, Kim, and Xie [2] (the LBL group) have derived analytical models of high gain FEL amplifiers operating in the linear regime that include 2-dimensional effects. Both models have been compared with the large numerical simulation codes, FRED and FELIX, and are found to agree with the simulations to within a few percent for beams for which 2dimensional effects are significant.. Thus one can base FEL design with confidence upon analytic theory rather than upon numerical simulation.

1 Lawrence Livermore National Laboratory, Livermore, CA 94550 and Dept. of Physics, UCLA.

Performed under the auspices of the Lawrence Livermore National Laboratory for the US Department of Energy under Contract No. W-7405-eng-48

2 Lawrence Berkeley National Laboratory, Berkeley, Ca 94720.

Work supported by the US Department of Energy under Contract No. DE-AC03-76SF00098 with the Lawrence Berkeley Laboratory.

3 Brookhaven National Laboratory, Upton, NY 11973. 
The analyses of Ref. [1] and [2], which are formulated for a beam with a "waterbag" distribution and for a Gaussian beam distribution respectively, have yielded scaling laws and associated universal graphs of FEL behavior. The waterbag model has the advantage of reducing to the correct, cold-beam limit; however, it probably overestimates performance for realistic beams with significant energy spread, emittance, and non-uniform phase space distributions. In this latter case, the Vlasov model of a Gaussian beam [2] provides a more prudent basis for FEL design. Although Ref. [1] and [2] have proved useful to many workers, their physical interpretation is obscured to some degree because both analyses are formulated in variables with lessthan-obvious physical significance. For this reason the 2-D theories (often incorrectly referred to as 3-dimensional) have not been employed as widely as should be the case.

Some years ago, a simple 1-dimensional analysis, with careful attention to the limits of validity of the one dimensional theory was given by Barletta and Sessler [3]. In particular, Ref. [3] introduced three dimensionless parameters, $f_{1}, f_{2}$, and $f_{3}$, that characterize the validity of the 1-dimensional, r-scaling of FELs derived by Bonifacio, Pellegrini, and Narducci [4]. The $f_{i}$ are defined in terms of the rms beam characteristics as:

$$
\begin{gathered}
\mathrm{f}_{1}=\frac{\varepsilon_{\mathrm{n}}}{\gamma(\lambda / 2 \pi)}, \\
\mathrm{f}_{2}=\frac{2 \varepsilon_{\mathrm{n}}}{\rho \mathrm{a}_{\mathrm{b}}^{2}\left(1+\mathrm{a}_{\mathrm{w}}^{2}\right)}, \\
\mathrm{f}_{3}=\frac{L_{\mathrm{G}}}{\mathrm{Z}_{\mathrm{R}}},
\end{gathered}
$$

where $e_{n}$ is the normalized emittance, $r$ the BPN scaling parameter, 1 the radiation wavelength, $g$ the relativistic factor, $a_{w}$ the dimensionless vector potential of the wiggler, $a_{b}$ the beam radius, $L_{G}$ the gain length for power, and $Z_{R}$ the Rayleigh range. In a careful restatement of arguments made by various workers through the years, 
Ref. [3] argued that validity of the 1-D theory (in the sense that 2-D effects only reduce the gain) requires that all the $f_{i} \leq 1$. Satisfying the resonance condition requires that $f_{2}=$ $\mathrm{f}_{1}{ }^{2} \mathrm{f}_{3}$; hence, the three criteria are not independent despite the apparent differences in the physical arguments used in their derivation. As Ref. [3] assumed that the energy spread in the beam was negligibly small, a more complete analysis should introduce an additional parameter, $f_{e}$, as a measure of the energy spread. One expects that $f_{e}$ should be small $(<1)$ for the cold-beam, 1-D results to apply.

The f-factors $f_{1}$ and $f_{3}$ are physically transparent in the sense that they allow for immediate physical interpretation. Therefore, they are easy to remember and to use. Can the more complete 2-D analyses of Ref. [1] and [2] be expressed in terms of $f_{1}, f_{3}$, and a transparent variable measuring the energy spread? The answer is yes. That the physically transparent variables completely describe the 2-dimensional state of the beam shows both the soundness of the physical arguments put forward in Ref. [3] and the underlying validity of the 1-dimensional analysis of the r-scaling of FEL performance first derived in Ref. [4]. In this sense we expect that if the 2-dimensional theories are expressed in terms of physically transparent variables, the resulting form of the theory will be easier to understand and to use.

In the next section we define the physically transparent ratios, $\mathrm{r}_{\mathrm{i}}$. These ratios are essentially the f-factors normalized to have unit value at the edge of the regime of applicability of the predictions of the 1-D theory. We then present model equations that represent a combined fit to features of both 2-D models. The combined fit aims at avoiding potential shortcoming of both models as applied to beams with realistic phase space distributions. To illustrate the consequences of the 2-D effects we present graphs of the linear growth rate in terms of the $r_{i}$. Our conclusion is that with the exception of the analysis of wiggler field errors and misalignment sensitivities (true 3-dimensional effects), the complete analysis of an FEL in the linear regime is in hand (thanks to the work of the BNL and LBL groups) and now in a form that is convenient to use. Indeed, 
for the bulk of the work of designing optical FELs (without waveguides), one can simply make all the $r_{i}$ close to unity and use the 1-dimensional theory of Ref. [4].

\section{Formulation}

Following the arguments given in Ref. [3], we introduce the ratios $r_{i}$. The first ratio is the normalized, full width of the energy spread,

$$
r_{1}=2 \frac{\Delta E}{\rho E},
$$

where the fractional energy spread, $\triangle E$, is a rms value and where $r$ is the BPN scaling parameter [4] for a planar wiggler, given by:

$$
\rho=\left[\frac{a_{w} \omega_{\mathrm{P}} \lambda_{\mathrm{W}} \mathrm{JJ}}{8 \pi \mathrm{c}}\right]^{\frac{2}{3}}
$$

In the formula $l_{w}$ is the wiggler wavelength, $a_{w}$ the dimensional vector potential of the wiggler strength, and $w_{p}$ is the relativistic plasma frequency of the drive beam. The Bessel function factor $\mathrm{J}$ is

$$
\mathrm{JJ}=\mathrm{J}_{1}(\xi)-\mathrm{J}_{0}(\xi)
$$

where

$$
\xi=\frac{a_{w}^{2}}{2\left(1+a_{w}^{2}\right)} \text {. }
$$

The second ratio is

$$
\mathrm{r}_{2}=\frac{4 \pi \varepsilon}{\lambda}
$$

where $e$ is the rms geometrical emittance of the drive beam, which is in terms of the normalized emittance, $e_{n} / g$. The third ratio is

$$
r_{3}=\frac{L_{G}}{Z_{R}},
$$

where $L_{G}$ is the e-foiding (gain) length for the power carried by the electromagnetic field as computed from the 1-dimensional theory. $L_{G}$ is given by the general expression, 


$$
L_{G}=\frac{\lambda_{w}}{4 \pi \rho \operatorname{Im}(2 \mu)},
$$

where $\operatorname{Im}(\mu)$ is the solution to the (cubic) eigenvalue equation fos the FEL instability written for the field intensity. In the cold-beam, 1-D limit, $\operatorname{Im}(\mu)=\sqrt{3} / 2$. The Rayleigh length, $Z_{R}$ is related to the rms beam radius, $a_{b}$, and to the optical wavelength, $l$, by

$$
\mathrm{Z}_{\mathrm{R}}=\frac{\pi \mathrm{a}_{\mathrm{b}}^{2}}{\lambda} \text {. }
$$

In terms of the $r_{i}$ we may evaluate the linear gain contours, $\operatorname{Im} \mu\left(r_{i}\right)$. The Gaussian beam model is a more conservative and probably more realistic model to use in design studies for cases in which two dimensional effects are important. Unfortunately the closed form, analytic formulae of Ref. [2] contain singularities for small values of $r_{3}$ and the model does not show the correct physical limit as the $r_{i}$ go to zero. For this reason we have modified the analytical formulae of Ref. [2] to eliminate the singular behavior in the limit of no diffraction and to give much better agreement with the waterbag model in the cold beam limit. In terms of the physically transparent variables, we find the following model equations (valid for $r_{3}>0.05$ ) for the combined fit to Gaussian beam in 2-D limit and waterbag model in the cold beam limit:

$$
\begin{gathered}
S=\frac{3^{1 / 4}}{2^{7 / 2}} r_{3}^{1 / 2} r_{1} \\
B=1.15 \frac{3^{3 / 4}}{2^{7 / 2}} r_{2} r_{3}^{3 / 2}+0.0028 \\
D=\frac{2^{5 / 2}}{3^{1 / 4}} r_{3}^{-1 / 2} \\
X=\operatorname{Ln}\left[\frac{2^{7 / 2}}{0.0035+3^{3 / 4} r_{3}^{3 / 2}}\right] \\
Y=41.34+3.69 X+3.62 X^{2}
\end{gathered}
$$




$$
\begin{gathered}
W=\left(0.33+0.095 r_{2}^{2 / 3}-0.015 r_{2}\right) S^{2}+2.18 S^{4}+70.9 S^{6} \\
U=\left(0.739+0.197 X+0.0175 X^{2}-0.00031 X^{3}\right) \\
R=0.03 r_{2}^{2}\left[1-\left(1+\exp \left(\frac{r_{2}-0.32}{0.06}\right)\right)^{-1}\right]+3^{3 / 2}\left(0.025 r_{2}^{2}+0.05 r_{2}^{4}+\frac{0.01}{64} r_{2}^{6}\right) r_{3}^{3} \\
\operatorname{Ln}\left(\frac{2 \mu}{D}\right)=-U\left\{1+\left[\frac{R}{8(0.17+0.0304 \ln B)}\right]+Y W\right\}^{1 / 2}
\end{gathered}
$$

A comparison of the combined model equations with the predictions of the waterbag and the Gaussian models for two cases with small diffraction is shown in Fig. 1. To assess the limits of validity of the 1-D model it is most useful to look at a plots of the gain surface, $\operatorname{Im}(\mu)$, as a function of the scaled emittance and diffraction. Two such plots for $r_{1}=0 \quad r_{1}=1$ are given in Fig. $2 a, b$. The behavior of the function $\operatorname{Im} \mu\left(r_{i}\right)$ for $r_{i}$ $<0.05$ has been suppressed. Note that even a small energy spread make the performance much more sensitive to emittance and diffractive effects. Fig. 3 shows the dependence of the gain, $\operatorname{Im} \mu\left(\mathrm{r}_{1}, \mathrm{r}_{2}\right)$, as a function of energy spread and emittance for two values of the diffraction parameter, $r_{3}$. While strong diffraction does not significantly reduce the gain for a cold, mono-energetic beam, it does make performance much more sensitive to emittance and energy spread.

The theory developed here can easily be extended to allow for conditioned beams, as has already been done in analytic form for the water bag model.[5] A graph of Im $\mu$ for a Gaussian beam with a "conditioned" energy distribution, Fig. 4a, when compared with the corresponding graphs for a beam without conditioning, Fig. 4 b, shows very clearly the advantage of using conditioned beams to suppress emittance, energy spread and diffraction effects. 
In optimizing an FEL design one may minimize the gain length; alternatively one might maximize the output power at saturation. Ref. [4] argues that the output power of the FEL at saturation can be expressed in terms of the scaling parameter $r$; i.e.,

$$
P_{\text {sat; 1-D }}=\rho P_{\text {beam }} \text {. }
$$

The corresponding expressions for the output power of the FEL at saturation given in Ref. [1] and [2] are not as easily interpreted. One can, however, rewrite these expressions in a form more readily understood in terms of the predictions of the 1dimensional theory. In particular the approximate expression of Ref. [2] reduces to

$$
P_{\text {sat; } 2-D}=\left(\frac{\operatorname{Im}(\mu)}{\sqrt{3} / 2}\right)^{4} \rho P_{\text {beam }} \text {. }
$$

Simulations indicate that the exact expression for the power at saturation in the presence of strong 2-D effects is far more complicated in its dependence on the $r_{i}$ than indicated in Eq. (22). If the figure of merit for the FEL optimization is the peak spectral brilliance, $B_{s}$, one must recall that the gain-broadened line width of the radiation is $r$. Consequently,

$$
B_{s} \propto \frac{P_{\text {sat }}}{\rho} \propto[\operatorname{Im}(\mu)]^{4} .
$$

This quantity always decreases with increased external focusing. One must be clear what figure of merit is to be optimized in the design of the free electron laser.

\section{Conclusions}

The results of the 2-dimensional theories of free electron laser amplifiers in the linear gain regime can be expressed in terms of the dimensionless, physically transparent ratios $r_{1}, r_{2}$, and $r_{3}$. The resulting dependences can be seen in the graphs of the gain eigenvalue which have been calculated on the basis analytic theories using a waterbag model [1], a Gaussian model [2], and a combined fit that yields preferred limiting behavior. One sees that 2-dimensional effects are alwrays "bad" in the sense that they reduce the 1-D gain length. Fortunately, in designing an FEL the deleterious 2-D effects 
can be eliminated by making the $r_{i}<1$ through a judicious choice of beam characteristics. Since making the $r_{i}$ small is difficult (and/or expensive), it is usually most practical to design an FEL with the $r_{i}$ close to, but less than, unity.

In practice, our prescription suggests that in designing an FEL one employs the lowest emittance gun one can obtain and, then, builds the lowest energy accelerator that allows $r_{2}$ to be of the order of unity for the wavelength of interest. One then checks that $r_{1}$ is of the order of unity. (If not, one either allows the energy spread to increase by obtaining more current from the gun or bunching the beam at an intermediate energy, or one decreases the energy spread in the beam by, for example, using a higher energy or lower current beam.) Finally one checks that $\mathrm{r}_{3}$ is near unity. If not, one decreases the gain length (for example by going to lower energy where $\mathrm{I}_{\mathrm{W}}$ is smaller), or in the opposite case, one can increase the focusing--beyond "natural focusing"--by the use of external magnetic elements or plasmas. At each point one must re-evaluate $r$ and iterate the procedure. In all cases one should be sure to "optimize" FEL with respect to the appropriate figure of merit for the application desired. For example, if the extremely high peak power is essential for the application, one may be forced to increase the beam energy much higher than our prescription would indicate. The price will be a much longer wiggler to reach saturation. Alternatively, one may also leave the beam parameters as is and taper the wiggler to increase the output power.

At this point one must also consider how the choice of beam characteristics will affect the alignment of the FEL and field tolerances in the wiggler. Unfortunately, there is not presently a complete analytic theory of such three dimensional effects for high gain FELs. In the absence of such a theory one might describe steering errors as leading to an equivalent emittance, which should then be kept less than $1 / 2 \pi$. Presently, one must resort to simulation for a sound, quantitative assessment at this stage of the design study. 


\section{Acknowledgments}

We acknowledge many useful conversations with Xwang-je Kim, Yong-Ho Chin and Ming Xie of LBL and Rodolfo Bonifacio of Un. of Milano during the course of our work. Their suggestions have lead us to enlarge the study beyond its original scope.

\section{References}

1. L.-H. Yu, S. Krinsky, and R. L. Gluckstern, Phys. Rev. Lett. 64, 3011 (1990).

2. Y.-H. Chin, K.-J. Kim, and M. Xie, LBL Report 30673 (Sept. 1991) and Proceedings of the FEL Conference 1991 (to Nucl. Inst. and Methods ).

3. W. A. Barletta and A. M. Sessler, "Radiation from Fine, Intense, Self-Focused Beams at High Energy", in High-Gain, High-Power Free-Electron Lasers: Physics and Application to TeV Particle Acceleration, North Holland (1989).

4. R. Bonifacio, C. Pellegrini, and L. Narducci, Optical Comm. 50, 373 (1984).

5.. A. M. Sessler, D. H. Whittum, and L.-H. Yu, Phys. Rev. Lett. 68, 309 (1992). 


\section{Figure Captions}

Figure 1. Comparison of waterbag model (dashed line), Gaussian model (light gray line) and combined fit (solid line).

Figure 2. The variation of FEL gain surface with scaled diffraction and scaled emittance as described in terms of physically transparent variables:

Fig. 2a) $r_{1}=0$, Fig.2b) $r_{1}=1.0$. Vertical and horizontal scales are linear.

Figure 3. Gain surface, $\operatorname{Im} \mu$, versus scaled emittance and energy spread for two values of the diffraction parameter, $\mathrm{r}_{3}$.

Figure 4. a) Gain contour, $\operatorname{Im} \mu$, for an emittance conditioned beam, b) Gain contour for an unconditioned beam with a scaled emittance $r_{2}=1$. 


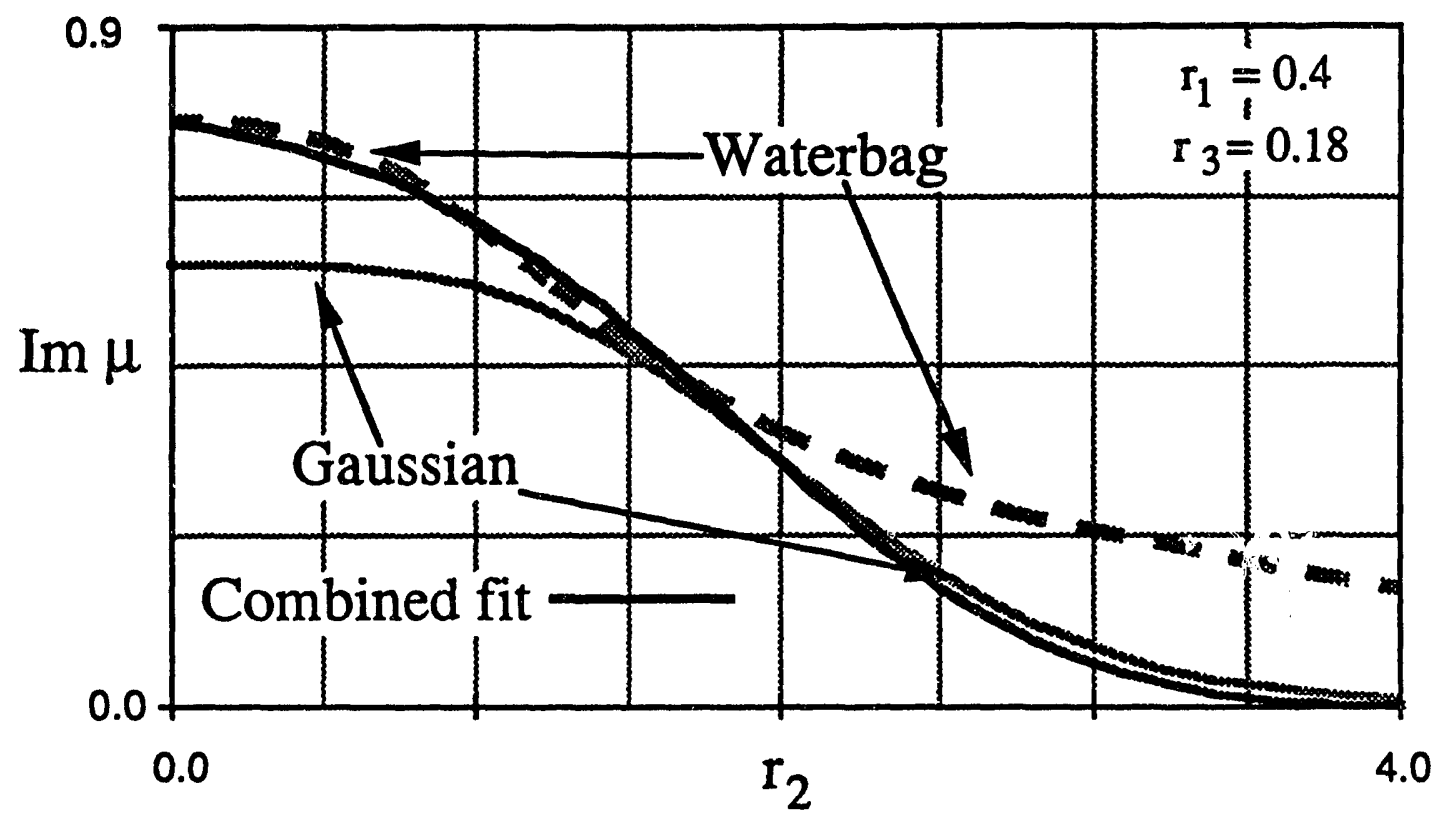

Fig. 1 

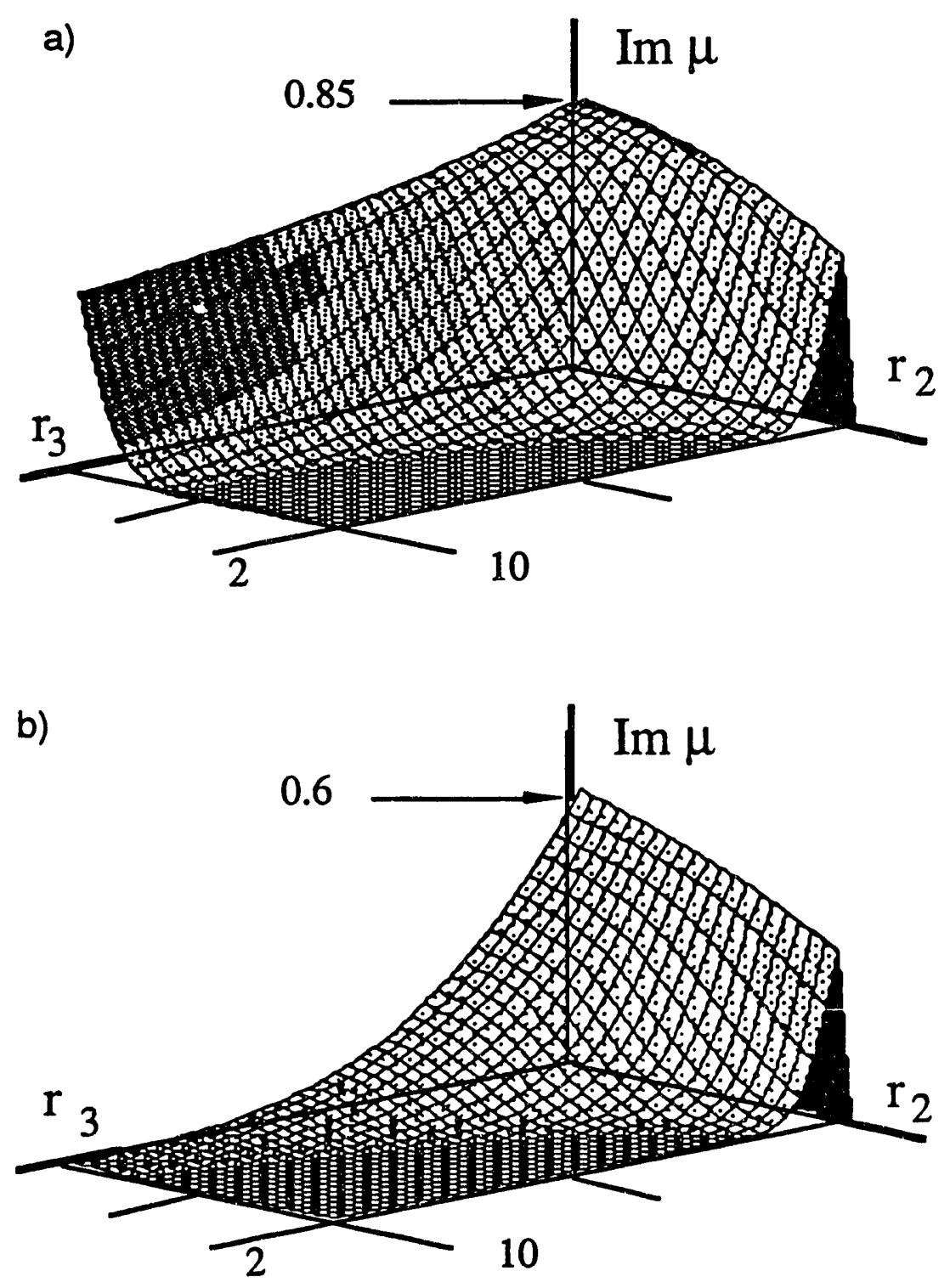

Fig. 2 


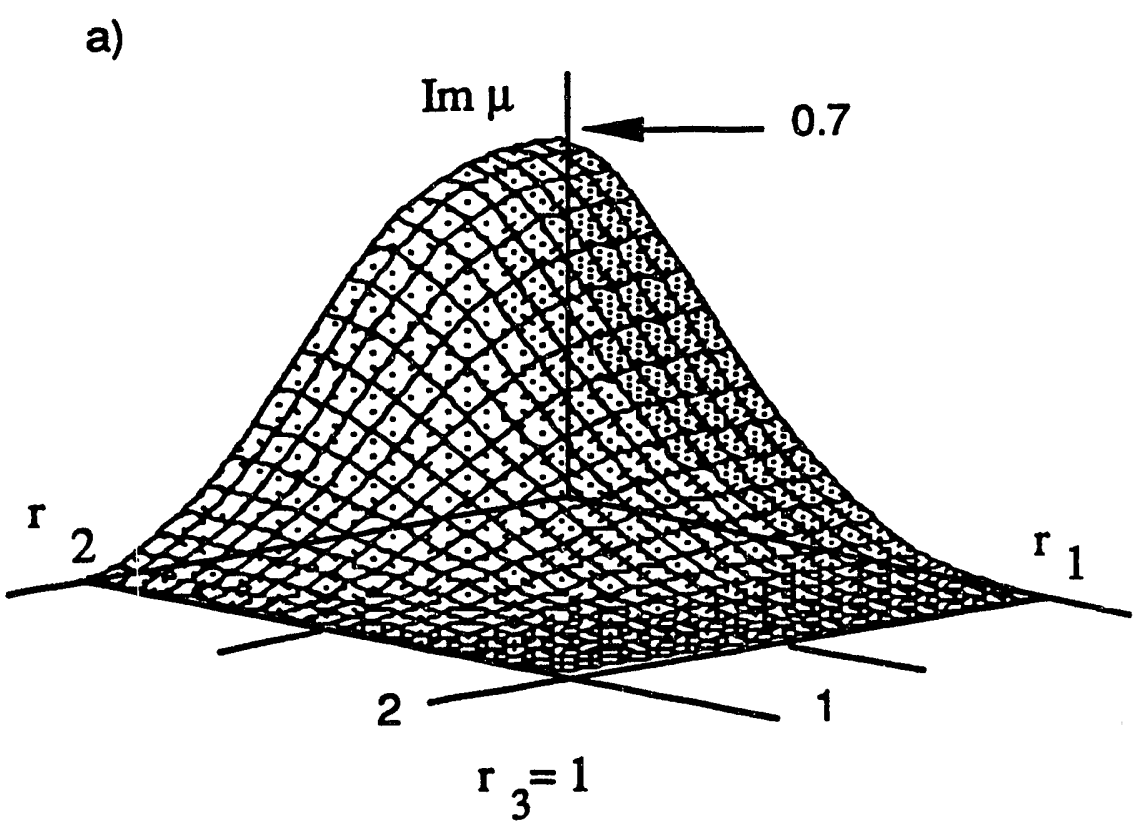

b)

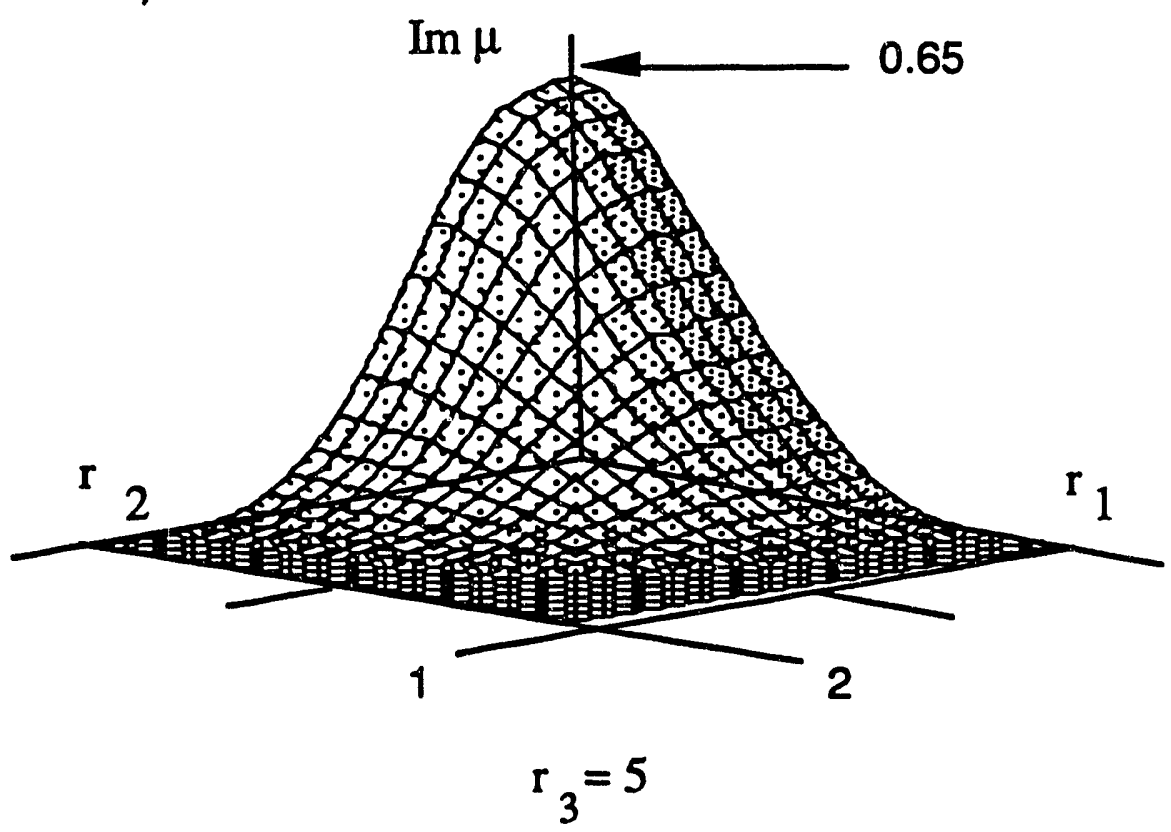

Fig. 3 


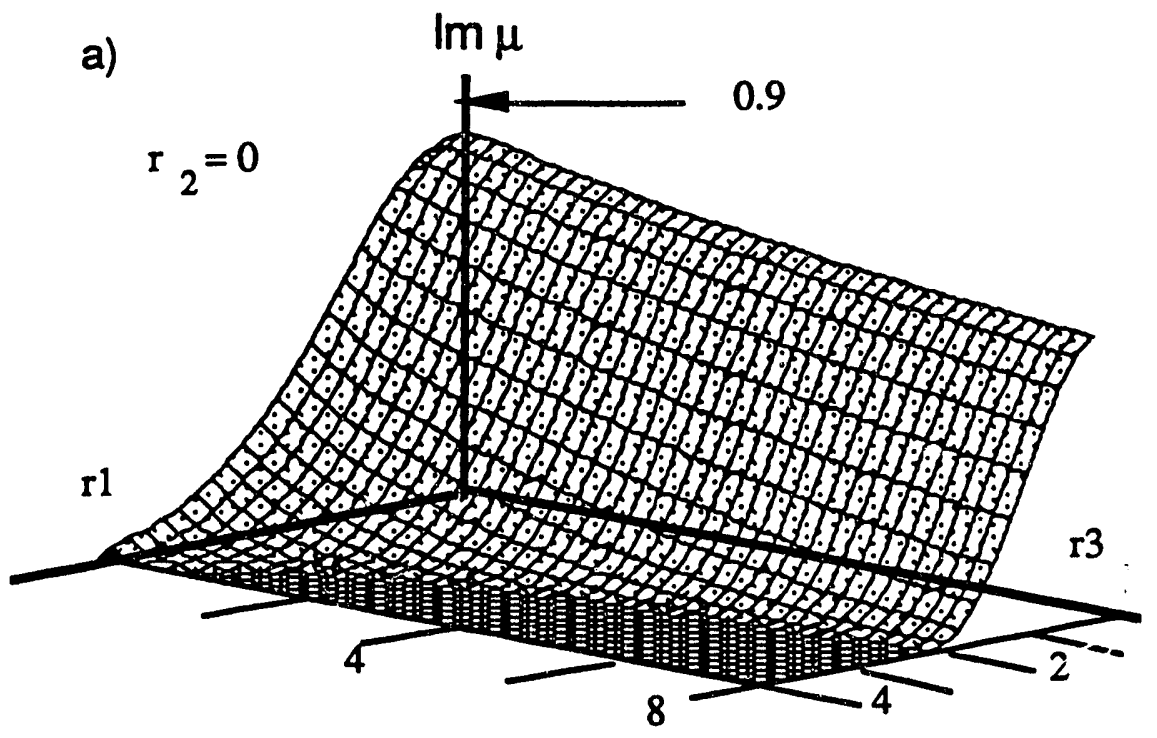

b)

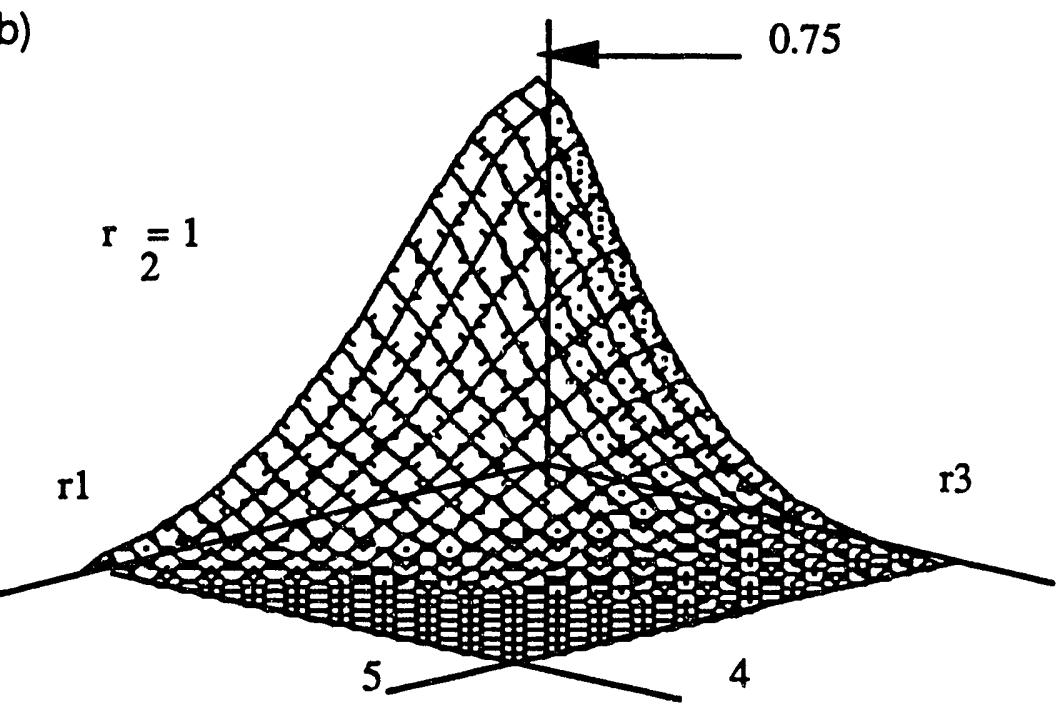

Fig. 4 

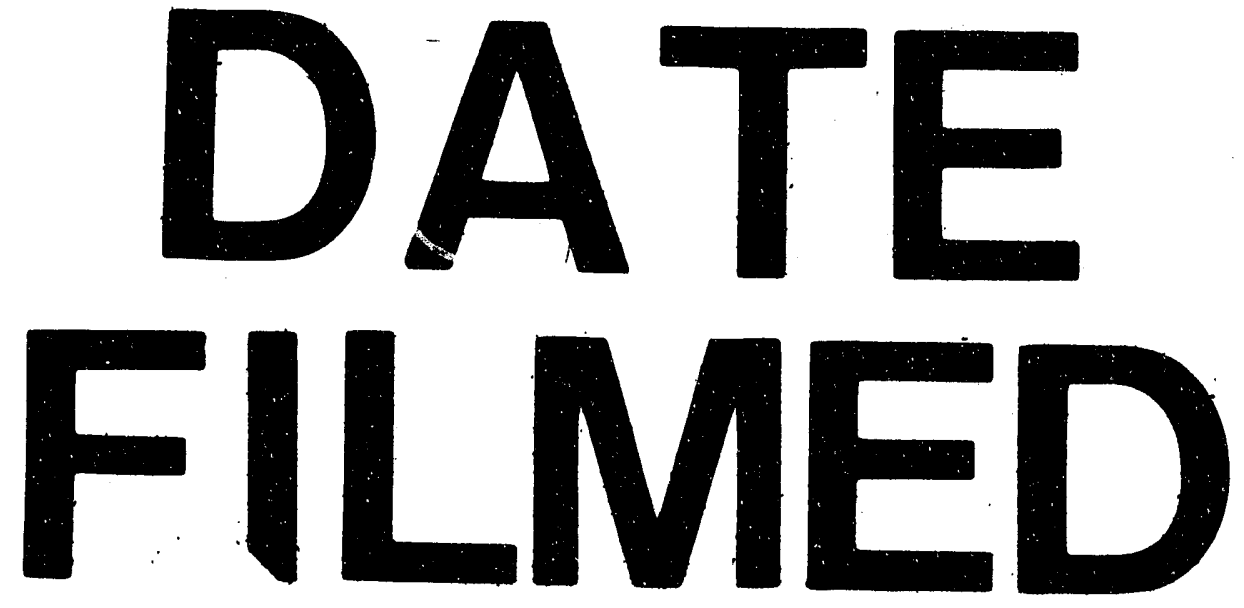

$01 / 20 / 93$ 
